\title{
A REAL-TIME REMOTE SENSING AND DATA ACQUISITION SYSTEM FOR A NUCLEAR POWER PLANT
}

\author{
KIHO KIM ${ }^{1}$, BUI VAN HIEU ${ }^{2}$, SEUNGHYUN BEAK ${ }^{2}$, SEUNGHWAN CHOI ${ }^{2}$, TAEHA SON ${ }^{2}$, JUNGKUK KIM ${ }^{2}$, \\ SEUNGCHUL HAN ${ }^{3}$, and TAIKYEONG JEONG ${ }^{2, *}$ \\ ${ }^{1}$ Korea Atomic Energy Research Institute \\ ${ }^{2}$ Dept. of Electronic Eng., Myongji University \\ ${ }^{3}$ Dept. of Computer Eng., Myongji University \\ ${ }^{*}$ Corresponding author. E-mail : ttjeong@mju.ac.kr
}

Received April 22, 2010

Accepted for Publication October 26, 2010

\begin{abstract}
A Structure Health Monitoring (SHM) system needs a real-time remote data acquisition system to monitor the status of a structure from anywhere via Internet access. In this paper, we present a data acquisition system that monitors up to 40 Fiber Bragg Grating Sensors remotely in real-time. Using a TCP/IP protocol, users can access information gathered by the sensors from anywhere. An experiment in laboratory conditions has been done to prove the feasibility of our proposed system, which is built in special-purpose monitoring system.
\end{abstract}

KEYWORDS : Real-time Remote Sensing, Nuclear Power Plant, Sensor and Sensor Interface Design

\section{INTRODUCTION}

Important structures such as bridges, nuclear containment buildings and dams, etc. must be ensured free-failure operation over the course of their lifetime. Thus, their operation status must be measured, reported, and evaluated over time by an independent system called a Structure Health Monitoring (SHM) system.

The system involves a set of sensors deployed in the structures to monitor their operation status. A Fiber Bragg Grating (FBG) sensor is a type of optical sensor which is often used in structural health monitoring. FBG sensors have many advantages such as light weight, electromagnetic immunity, resistance to harsh environments and easy multiplexing [1][2]. The only drawback of applications based on FBG sensors is high cost because of the expense of the associated interrogation system. Recently, the price of such systems has been reduced by new research [3].

One essential requirement of the SHM system is to acquire all sensor values in real-time and remotely. On this front, there have been some systems developed for

\footnotetext{
* This work was supported by the Research \& Development Program of the National Research Foundation of Korea (NRF), funded by the Ministry of Education, Science \& Technology (MEST), Grant code: 2010-0001139.

(Inquiries to the Corresponding author : T. Jeong, E-mail : ttjeong @ mju .ac.kr, $\mathrm{Ph}:+82-31-330-6775)$
}

real-time monitoring of machines, patient health, and building structures, etc [4][5][6]. One recent study reporteda newly developed system by P.J. Bock which can acquire data from $t$ remote locations in real-time [7].

In this paper, we present a real-time remote data acquisition system for a Nuclear Power Plant (NPP). The system uses FBG sensors to measure pressure on the shield of the NPP by sensing strain on the shield. The system can process up to 40 FBG sensors. The strain values from the sensors are collected in real-time and stored on a server which is located at the NPP site. This server provides sensor data to clients over the Internet. Clients can thus acquire sensor values remotely and in real-time.

The remainder of this paper is organized as follows. In Section II, we present the overall system structure and its components. In Section III, we discuss data communication between the server and clients over the Internet. In Section IV, a prototype system is described and its results are presented. The last section is the conclusion.

\section{SYSTEM STRUCTURE}

The overall system structure is shown in Fig. 1 . The first component of the system is a set of sensors. The sensors used in the system are FBG sensors. FBG sensors sense strain changing on the shield of the NPP. The second 
component of the system is an optical interrogator. The interrogator receives optical signals from sensors and transforms them to the corresponding strain on the sensors. The strain values are transferred to the third component, a server, which connects directly with the interrogator. Clients can connect with the server over the Internet. Using the connection with the server, clients can access sensor values in real-time over the Internet.

The rest of this section will describe the components of the system in more detail.

\subsection{FBG Sensors}

An FBG sensor is a type of optical sensor. FBG sensors reflect a component of transmitted light and transmitted all other optical sensors. The wavelength of the reflex light is determined by the structure of the FBG sensor, the strain applied, and the temperature around the FBG sensors. Hence, an FBG sensor can sense changes in temperature and strain directly.

An FBG sensor is calibrated before use. At the normal temperature and without strain, the wavelength of the reflex light is determined by the structure of the FBG sensor itself [2]. The wavelength is determined by Equation (1) :

$\lambda_{r}=2 n \Gamma$

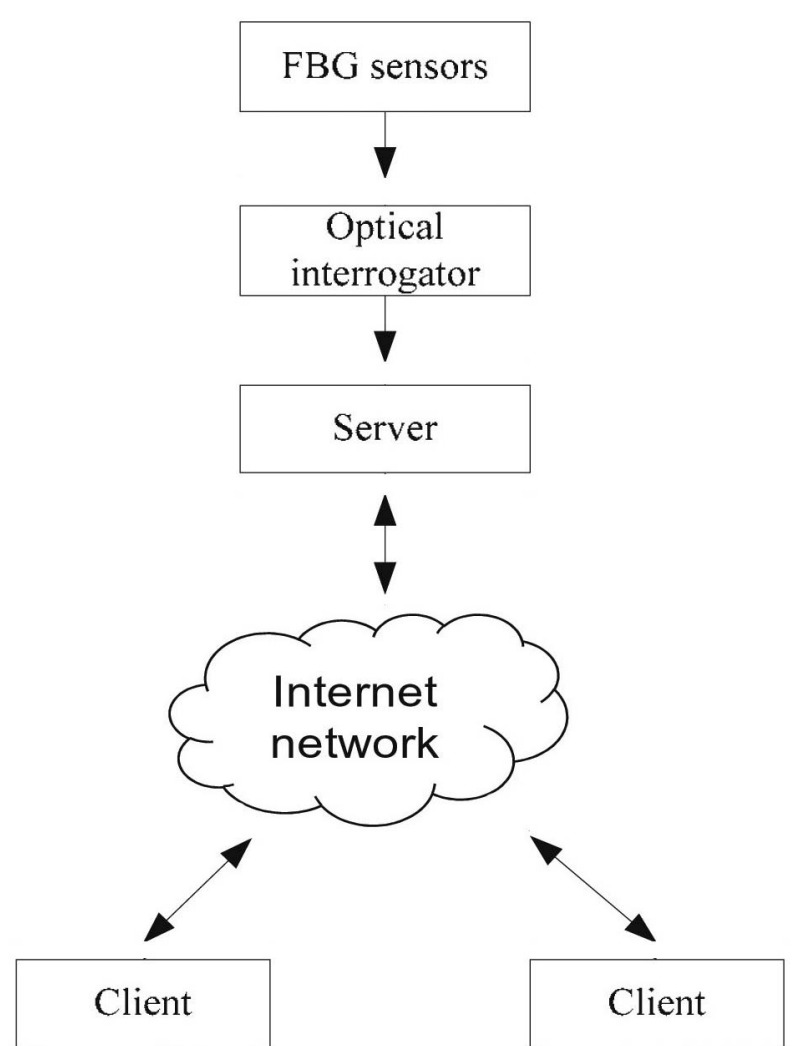

Fig. 1. Overall Structure of the Remote Acquisition System where $\lambda_{r}$ is the wavelength of the reflex light, $n$ is the effective index of the core of the fiber, and $\Gamma$ is the grating pitch.

From the calibrated value, strain applied to the FBG sensor can be sensed by changes in the wavelength of the reflex light. When strain is applied to the sensor, the changes to the wavelength of the reflex light are determined by Equation (2):

$$
\partial \varepsilon=\frac{\partial \lambda_{r}}{0,78.10^{-6} \lambda_{r}} \quad\left(\mu \varepsilon^{-1}\right)
$$

where $\partial \varepsilon$ is the amount of strain change, $\partial \lambda_{r}$ is the amount of wavelength shift of the reflex light, and $\lambda_{r}$ is the wavelength of the reflex light of the FBG sensor under conditions of zero strain.

\subsection{Optical Interrogator}

The optical interrogator provides light for the FBG sensors, receives reflex light from the FBG sensors, calculates the wavelength shift, and transfers wavelength information to a PC. The internal structure of the interrogation system is shown in Fig. 2 .

The light source provides light for the sensors. An optical switch distributes light equally from the light source to four optical connectors. Optical fibers including FBG sensors connect with the interrogation system by optical connectors. Reflex light from the FBG sensors propagates back to the connectors, and then goes to the optical switch. The optical switch and coupler route

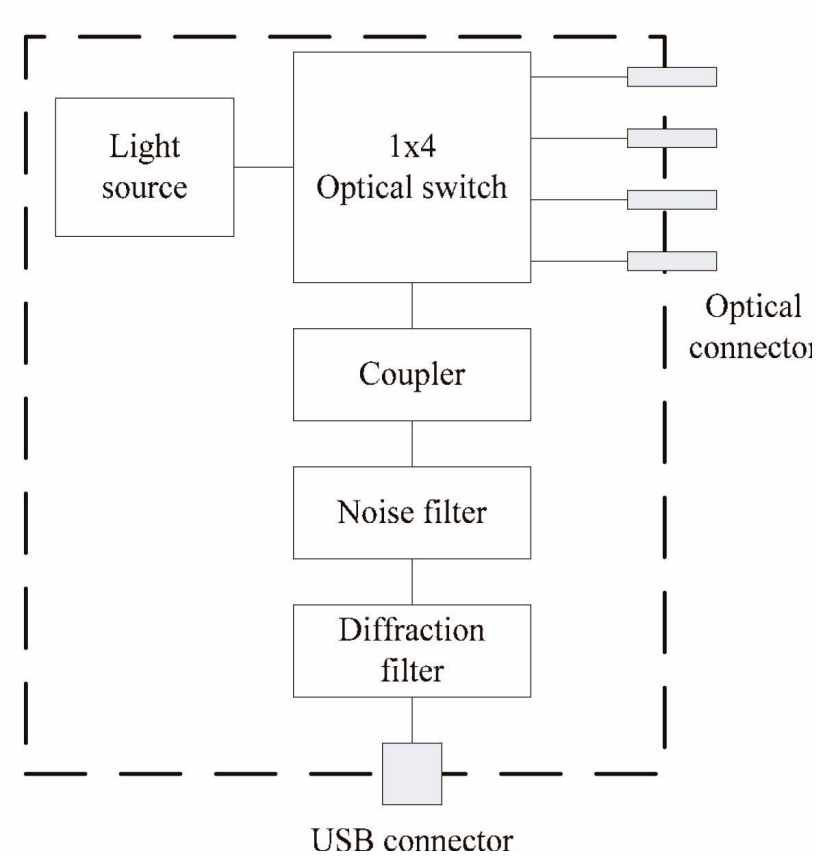

Fig. 2. Internal Structure of the Optical Interrogator 
reflex light to the noise filter. The noise filter removes noise and then the diffraction filter determines the wavelength of the reflex lights. Using the wavelength information, the wavelength shift or strain can be calculated. Wavelength information is transferred to a server via the Universal Serial Bus (USB) protocol.

The interrogator supports 4 (four) optical connectors or channels. The diffraction filter can detect 10 different wavelengths for each channel as long as the reflex wavelengths of the FBG sensors do not overlap. Consequently, the interrogator can support up to $40 \mathrm{FBG}$ sensors.

\subsection{Server}

The server communicates with the interrogator over USB protocol, collects sensor data, displays data, stores sensor data, and sends data to clients. The server functions are implemented in Labview.

After collecting the data from the interrogation system, the values of the FBG sensors can be monitored at the server. The server program supports four different views to display sensor values, as shown in Fig. 3 . In this case, the data transmission is made by a data acquisition system, at approximately $3 \mathrm{Gbps}$, and each channel speed is also $2.54 \mathrm{MHz}$.

The 'Dynamic' view on the server program draws the strain values of an FBG sensor in time. The 'Fourier' analysis view displays the Fourier analysis of a reflex light [8]. The 'Strain' view displays the reflex wavelength and current strain of all FBG sensors. The 'Wavelength' view displays the reflex wavelengths of all FBG sensors [9]. The particular FBG sensor displayed in the 'Dynamic' view or 'Fourier' analysis view can be specified by the user.

The data is also stored at the server every three hours for later access as well as for analysis of the long-time operation of the structure. When clients request sensor data, the data is transmitted to clients over the Internet by using TCP/IP protocol.

\subsection{Client}

'Client' is a program with a graphical user interface (GUI) that is easy to install on a computer running the

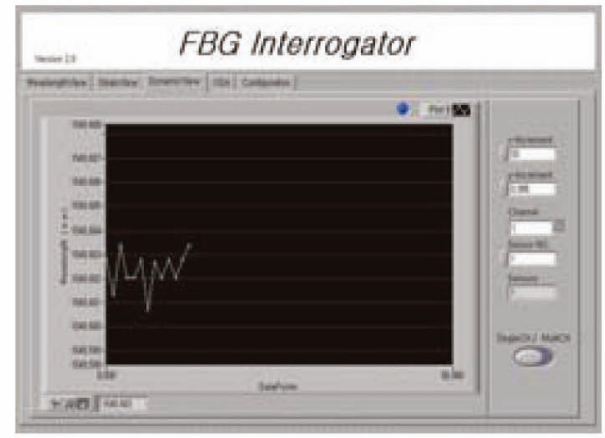

(a)

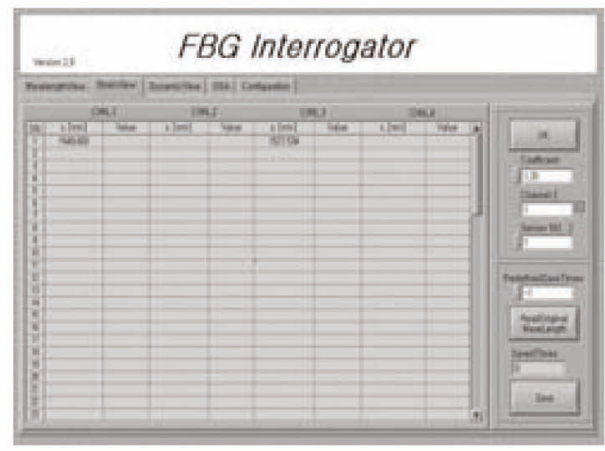

(c)

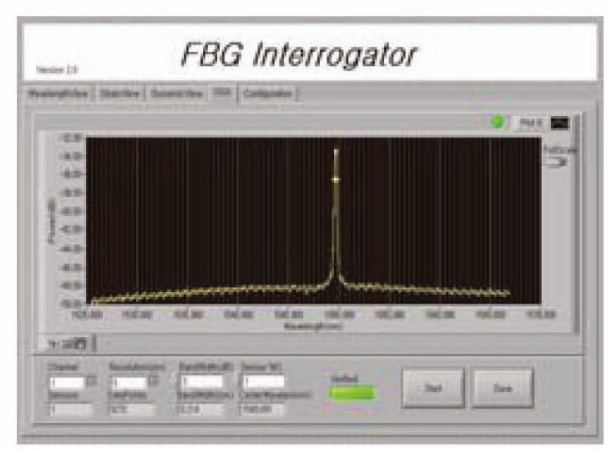

(b)

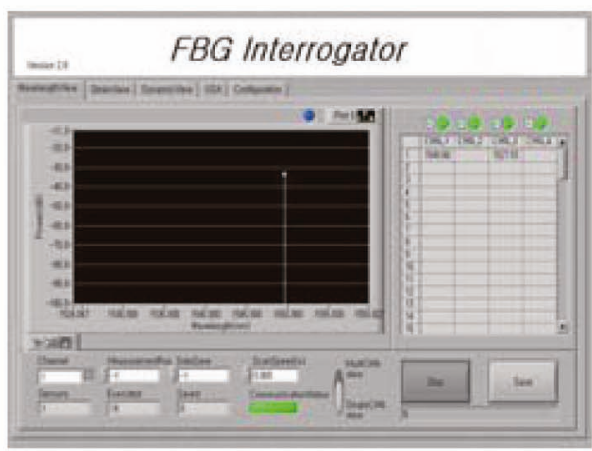

(d)

Fig. 3. Different Views of FBG Sensor Values at Server: (a) Dynamic View (b) Fourier Analysis View (c) Strain VIew, and (d) Wavelength View 
Windows operating system. Client can connect to the server over the Internet, can receive real-time data on monitored factors such as strength, can display the data in two forms, tabular form and graph, and can alarm the user when there is an abnormal condition in the monitored values. It is noted that Labview, a software design tool, is a functional tool for implementation of both the Server and the Client.

\section{DATA COMMUNICATION}

\subsection{Communication Diagram}

With a monitoring system, the correctness of sensor values must be guaranteed. Two protocols for transferring data over the Internet are the user datagram protocol (UDP) and the transmission control protocol (TCP). UDP transfers data unreliably, whereas TCP transfers data in a reliable manner [10]. Therefore, we use TCP for data communication in our system.

A data communication diagram for the proposed system is shown in Fig. 4. There are three entities involved in the communication system: the interrogator, server, and clients.

Firstly, the interrogator establishes connections with the server by sending a 'Request' message. If the server accepts the connection, it will confirm by sending an 'Accept' message to the interrogator. Thereafter, the interrogation system collects data from all channels and sends it to the server periodically, i.e., at one second intervals. To terminate the connection, the interrogation system sends a 'Termination' message.

Secondly, each Client program being used to monitor sensor values in real-time must establish a connection with the server. Similarly to the interrogation system, each Client sends a 'Request' message and receives an 'Accept' message if the server accepts the connection. The server will deny the connection if the identify process does not recognize the client.

After the connection between a Client and the server is established, the server broadcasts data to the Client whenever it receives data from the interrogator. When the interrogator terminates the connection with the server, the server sends a 'Termination' message to any Clients.

\subsection{Message Format}

For communication, two message formats are used: command message and data message format.

The command message format is used to establish and terminate connections. The data message format is used to transfer sensor values between components. The command and data message formats are shown in Fig. 5 .

Because communication messages are short, message synchronization can be accomplished easily using the

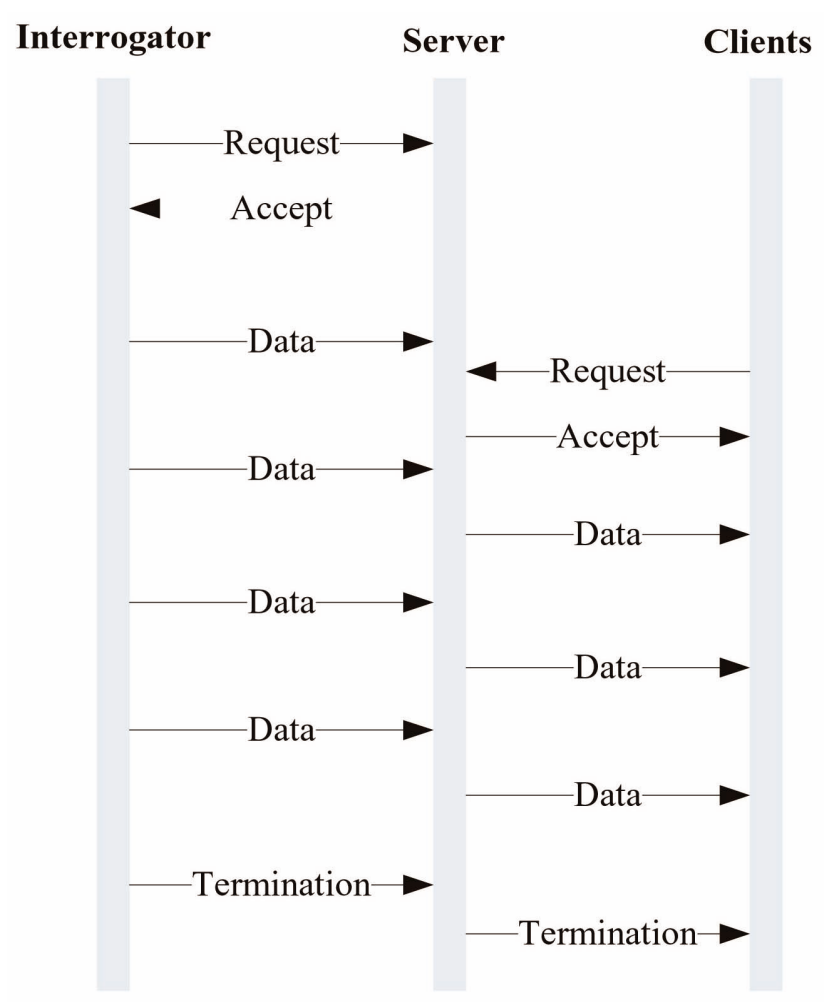

Fig. 4. Data Communication Diagram

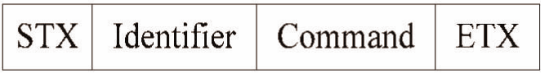

(a)

\begin{tabular}{|l|l|l|l|l|l|}
\hline STX & Identifier & Command & Channel & Data & ETX \\
\hline
\end{tabular}

(b)

Fig. 5. Message Formats: (a) Command Message, and (b) Data Message

two synchronization symbols STX and ETX. The meanings of fields in the structure are listed below.

- STX : signals the beginning of a message

- Identifier : identifies the component sending a message (e.g. the interrogation system, server or particular client)

- Command : specifies the type of message (e.g. a command request, an accept message, or a termination message)

- Channel : for a data message, this field specifies the channel

- Data : the values from sensors

- ETX : signals the end of a message 


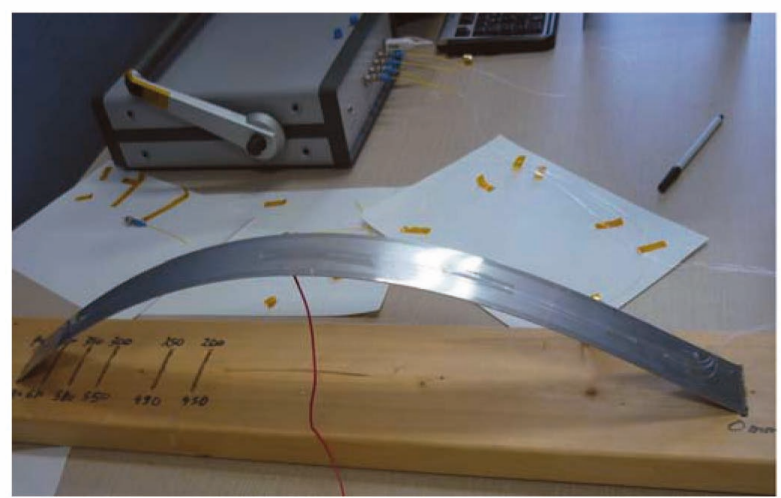

Fig. 6. An Illustration of the Experiment Structure

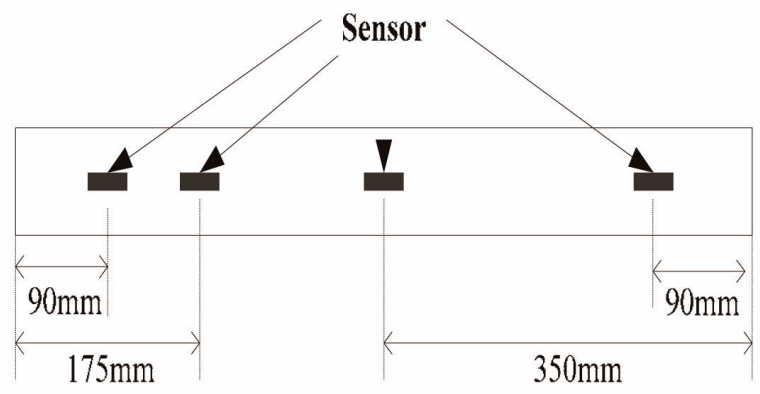

Fig. 7. The Deployed Position of FBG Sensors On the Aluminum Beam

\section{RESULTS AND DISCUSSION}

We tested the system in laboratory conditions. An illustration of the testing structure is shown in Fig. 6 . The structure is an aluminum beam fixed on a wooden support. The beam is $700 \mathrm{~mm}$ in length and $60 \mathrm{~mm}$ in width. The curvature of the aluminum beam can be changed by changing the position at which it is fixed. This makes the strain change and simulates the change of strain in a bridge under real conditions. Four (4) FBG sensors are deployed along the beam. The position of the FBG sensor is shown in Fig. 7 .

As discussed above, the interrogation system measures sensor values and transfers them to the server, and then the server broadcasts them to Clients. Fig. 8 is a screen capture of a Client monitoring the real-time value of FBG sensors. The values of the sensors are presented in the tabular form at the bottom of the window. The values over time are drawn above the tabular form. We applied a load at $40 \mathrm{sec}$ and $70 \mathrm{sec}$ and released the load at $95 \mathrm{sec}$. Changes in the sensor values correspond with changes in the load, as shown in the figure.

The values displayed on the client side are exactly the values on the server. This means the sensor values are correctly acquired remotely in real-time.

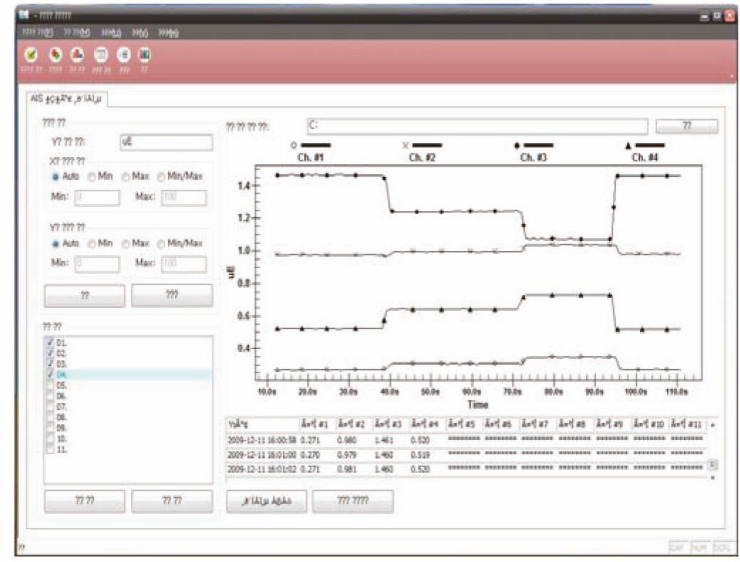

Fig. 8. Screen Capture of Real-time Remote Monitoring System

\section{CONCLUSIONS}

In this paper, we have described a real-time remote data acquisition system that can be used to gather FBG sensor values for NPPs. The acquisition system uses TCP/IP protocol to deliver data over the Internet. The system supports up to $40 \mathrm{FBG}$ sensors and it can be easily extended by adding interrogation systems. The system has been tested in laboratory conditions. The results prove that this system is suitable for monitoring FBG sensor values in real applications.

\section{ACKNOWLEDGEMENTS}

This work was also supported by an NRF grant funded by the Ministry of Education, Science \& Technology (MEST), No.2009-0069991. This work is also partially supported by the Korean government's MKE under grant No. I-2010-1-012 of the ETEP. This work was further supported by the 2010 research fund of Myongji University in Korea. The circuit was designed at the IC Design Center.

\section{REFERENCES}

[ 1 ] A. D. Kersey, M. A. Davis, H. J. Patrick, M. Leblanc, K.P. Koo, C. G. Askins, M. A. Putnam, and E. J. Friebele, "Fiber grating sensors," Journal of Lightwave Technology, vol. 15, pp. 1442-1463, August 1997

[2 ] W. W. Morey, G. Meltz, and W. H. Glenn, "Fiber bragg grating sensors," in Proc. SPIE Fiber optic and Laser sensors VII, vol. 1169, pp. 98, 1989

[3 ] A. Kerrouche, W.J.O. Boyle, T. Sun, and K.T.V. Grattan, "Design and in-the-field performance evaluation of compact FBG sensor system for structural health monitoring applications," Journal of Sensor and Actuators A, vol. 151, pp. 107-112, 2009

[4] M. Mori, M. Fujishima, M. Komatsu, B. Zhao, and Y. Liu, "Development of remote monitoring and maintenance system for machine tools," CIRP Annals - Manufacturing 
technology, vol.57, pp. 433-436, 2008

[ 5 ] M. Dey, and H.D. Pujara, "Real-time acquisition and remote monitoring of steady state tokamak (SST-1) diagnostics data," in Proc of the $24^{\text {th }}$ Symposium on Fusion technology, vol. 82 , pp. 1198-1202, 2007

[6] W. Xiaohong, and L. Li, "The design of remote medical monitoring system based on sensors and GPRS," in Proc. of Information technology and applications IFITA '09, vol. 3, pp. 516-519, 2009

[ 7 ] P. J. Bock, S. Majumdar, and W. J. Bock, "Internet-based distributed data acquisition system for fiber-optic sensors," IEEE Trans. Instrument and Measurement, vol. 56, no. 1, pp. 32-38, 2007
[ 8 ] Y. Kim, J. Lee, Y. Kim, and J. Kim, "A Highly Sensitive Humidity Sensor Using a Modified Polyimide Film," Journal of Semiconductor Technology and Science, vol.4, no.2, pp.128-132, 2004

[9] H. Jabbar, S. Lee, S. Choi, S. Beak, S. Yu, and T. Jeong, "A Novel Sensing Method and Sensing Algorithm Development for a Ubiquitous Network," MDPI, Sensor, vol. 10, no. 9, pp. 8129-8142, September 2010.

[10] S. Chang, J. Park, K. Won, and H. Shin, "Design of a 2.4GHz Fully Differential Zero-IF CMOS Receiver Employing a Novel Hybrid Balun for Wireless Sensor Network," Journal of Semiconductor Technology and Science, vol. 8, no.2, pp.143-149, 2008 